nary indicators fail. In the first method an index of the excess of ammonia is obtained by shaking out with chloroform and by titrating the chloroform with a dilute acid. In the second method the rise in temperature due to the heat of neutralization is observed as the titration proceeds, the end point being at the break in the heating curve. Both these methods are simpler than the method formerly proposed where the conductivities of solutions must be determined at constant temperature.

DEPARTAENT OF CHEMTSTKL,

UNIVERSTTY OF NORTH CAROLLNA,

Chafer, Hill, $\mathrm{X} . \mathrm{C}$.

[CONtribution from the Chemical, Laboratory of the OhIo State University.]

\title{
THE PREPARATION AND THE OXIDATION OF STYROLENE
} ALCOHOL.

By Wa. Lloyd Evans and lou helen Morgan. Received October 31, 1912.

The work presented in this report was undertaken with two distinct purposes in view. First, to determin the mechanism of the oxidation reaction of styrolene alcohol with any given reagent, $i$. e., which one of the five theoretical possibilities this reaction follows; and, second, as a result of these studies, to establish, if possible, the experimental conditions whereby mandelic aldehyde might be isolated as an intermediate oxidation product. From the well known experiments of $\mathrm{Nef}^{1}$ with mandelic aldehyde acetate, it was to be expected that if the free aldehyde was isolated at a temperature approaching $100^{\circ}$, it would immediately rearrange to benzoyl carbinol, the oxidation chemistry of which is understood. $^{2}$ The experiments of Wohl and Lange ${ }^{3}$ and more recently those of Kranz ${ }^{4}$ with lactic aldehyde seem to indicate that this substance is sufficiently stable at ordinary temperatures to be easily identified. In fact, Kranz reports it to be a white crystallin compound melting at $10 \mathrm{I}-\mathrm{IO} 2^{\circ}$.

The dissociation of styrolene alcohol in the process of oxidation may be regarded as taking place in any one or all of the following ways: ${ }^{5}$

I. $\mathrm{C}_{6} \mathrm{H}_{5}$. $\mathrm{CHOH} . \mathrm{CH}_{2} \mathrm{OH} \longrightarrow \mathrm{C}_{6} \mathrm{H}_{5} \cdot \mathrm{CHO}+\mathrm{CH}_{3} \mathrm{OH}$.

II. $\mathrm{C}_{6} \mathrm{H}_{5}$. CHOH. $\mathrm{CH}_{2} \mathrm{OH} \longrightarrow \mathrm{C}_{6} \mathrm{H}_{5}, \mathrm{CH}_{2} \mathrm{OH}+\mathrm{H}_{2} \mathrm{CO}(\mathrm{CHOH})$.

III. (a) $\mathrm{C}_{8} \mathrm{H}_{5} \cdot \mathrm{CHOH} \cdot \mathrm{CH}_{2} \mathrm{OH} \rightarrow \mathrm{C}_{6} \mathrm{H}_{5} \cdot \mathrm{C} / \mathrm{CH}_{2} \mathrm{OH}+\mathrm{H}_{2} \mathrm{O}$.

${ }^{1}$ Ann., 335, 266.

${ }^{2}$ Work is now being carried on in this laboratory by Mr. C. R. Parkinson and myself with a view of synthesizing mandelic aldehyde and studying its properties. W. L. F.

${ }^{3}$ Ber, 4 4, 3612.

- Chemicke Listy, 5, 323-7, C. A., 6, 739 .

- Compare This Jovrnal, 34, r Ior. 
(b)

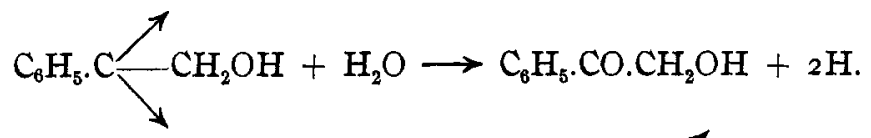
IV. (a) $\mathrm{C}_{6} \mathrm{H}_{5}$. CHOH.CH $\mathrm{CH}_{2} \mathrm{OH} \mathrm{C}_{6} \mathrm{H}_{5}$. CHOH.CH $\searrow+\mathrm{H}_{2} \mathrm{O}$.

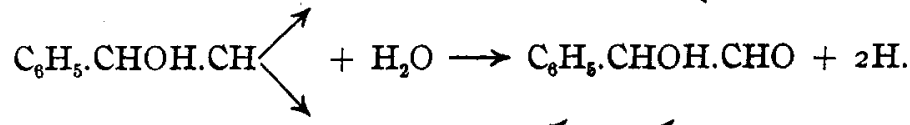

v. (a) $\mathrm{C}_{6} \mathrm{H}_{5} \cdot \mathrm{CHOH} \cdot \mathrm{CH}_{2} \mathrm{OH} \rightarrow \mathrm{C}_{6} \mathrm{H}_{5} \cdot \mathrm{C}_{\searrow} \nearrow^{\nearrow} \mathrm{CH}^{\prime}+{ }_{2} \mathrm{H}_{2} \mathrm{O}$.

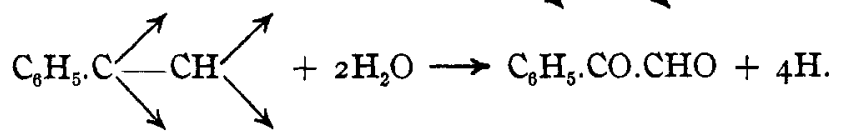

If styrolene alcohol dissociates in its oxidation phenomena in accordance with equations (I) and (II), then benzoic acid would be one of its final oxidation products. If the reaction proceeds in accordance with equation (III) then the oxidation products would be the same as those of benzoyl carbinol with the same reagent. If mandelic aldehyde is the first oxidation product, then mandelic acid and its subsequent oxidation products would be present; or, those obtained by the dissociation of mandelic aldehyde into benzaldehyde and formaldehyde in the same manner as proven below for benzoyl carbinol. Finally, if both the primary and secondary alcohol groups of styrolene alcohol are oxidized simultaneously, then benzoylformaldehyde, discovered by Von Pechmann ${ }^{1}$, would be formed. This substance would rearrange to mandelic acid if alkalies were present, and in their absence, benzoic acid and carbon dioxide would be formed. It is evident, therefore, that the nature of the reaction products formed will be determined in some measure by the oxidizing agents used. Zincke ${ }^{2}$ found in his experiments on the oxidation of styrolene alcohol, that benzaldehyde, formaldehyde, and formic acids were obtained with chromic acid, and with potassium permanganate ${ }^{3}$ benzaldehyde was obtained quantitatively when the reaction was carefully controlled. With nitric acid, he obtained benzoyl carbinol (80-90\% yield) and by longer action, benzoylformic acid was the product. With the exceptions noted, no quantitative data were given.

Zincke explained the mechanism of the oxidation reactions of styrolene alcohol by the two following equations:

(a) $\mathrm{C}_{6} \mathrm{H}_{5} \cdot \mathrm{CHOH} \cdot \mathrm{CH}_{2} \mathrm{OH}+\mathrm{O} \longrightarrow \mathrm{C}_{6} \mathrm{H}_{5} \cdot \mathrm{CHO}+\mathrm{H}_{2} \mathrm{CO}+\mathrm{H}_{2} \mathrm{O}$,

1 Ber., 22, 2557.

2Ann., 216, 303 .

${ }^{3}$ Compare Henderson and Gray, J. Chem. Soc., 85, ro43. 
i. e., benzaldehyde and formaldehyde may oxidize to benzoic and formic acids, respectively, and the latter finally to carbon dioxide and water;

(b) $\mathrm{C}_{6} \mathrm{H}_{5} \cdot \mathrm{CHOH} \cdot \mathrm{CH}_{2} \mathrm{OH}+\mathrm{O} \longrightarrow \mathrm{C}_{8} \mathrm{H}_{5} \cdot \mathrm{CO} \cdot \mathrm{CH}_{2} \mathrm{OH}+\mathrm{H}_{2} \mathrm{O}$

$$
\mathrm{C}_{8} \mathrm{H}_{5} \cdot \mathrm{CO} \cdot \mathrm{CH}_{2} \mathrm{OH}+\mathrm{O}_{2} \rightarrow \mathrm{C}_{8} \mathrm{H}_{5} \cdot \mathrm{COCOOH}+\mathrm{H}_{2} \mathrm{O} \text {, }
$$

i. e., benzoyl carbinol is an intermediate reaction product.

The plan followed in this work was to study each equation separately with each oxidizing agent used, $i . e$, to subject each possible dissociation product to exactly the same conditions of experimentation as those surrounding the oxidation of styrolene alcohol. As a result of these studies, it was hoped by a process of elimination to be able to give the exact course of the oxidation reaction for any given reagent. Stronger oxidizing agents were selected in the beginning, in order to ascertain what the end products might be under the conditions chosen. As these were better understood, milder oxidizing agents were employed, owing to the well known stability of some of the possible intermediate products towards them. This paper constitutes a preliminary report of the study of styrolene alcohol being made in this laboratory. The results of the experiments thus far made with the reagents given are briefly these:(I) Styrolene aleohol is best prepared by the hydrolysis of styrolene diacetate, which is in turn prepared most satisfactorily from styrolene dibromide and fused lead acetate. (2) Styrolene alcohol is oxidized with potassium permanganate alone or in the presence of alkalies to benzoic acid as one of its reactions products. Since benzoylformic acid is not an oxidation product in the presence of alkali, the reaction, in effect, proceeds in accordance with equations (I) or (II). (3) With potassium ferricyanide the oxidation in effect follows equations (I) or (II), since mandelic acid is not found as a reaction product. (4) With silver oxide, in the presence of alkali, styrolene alcohol is oxidized in accordance with equations (III) or (IV) or both at $60^{\circ}$. (5) With silver oxide in the absence of alkali, it is possible that the reaction proceeds entirely in accordance with equation (III) at about $20^{\circ}$. (6) Benzoylcarbinol is formed by the oxidation of styrolene alcohol by alkalin bromine solutions. (7) Aqueous solutions of copper salts are without any marked action on styrolene alcohol even at $100^{\circ}$.

\section{Experimental.}

Preparation of Styrolene Dibromide.-Zincke prepared styrolene dibromide by two different methods: ( $\mathrm{I}$ ) the action of bromine on hot ethyl benzene, by which procedure he obtained 1150 grams (theory $=125^{\circ}$ grams, therefore $92 \%$ ) from 500 grams of the hydrocarbon ${ }^{1}$ and (2) by the action of 17 parts of bromine upon Io parts of styrolene dissolved in about 20 parts of pure ether. By this method, he found the yield of

' Zincke, Ann., 216, 288; Radziszweski, Ber., 6, 493. 
the dibromide to be nearly quantitative if small amounts of materials were used. For the purpose of these experiments, it was found more convenient to make the following adaptation of method (2): To a solution of 48.5 grams ( $\mathrm{I} \mathrm{mol}$.) of freshly distilled styrolene in $400 \mathrm{cc}$. of pure ether, were added 126.8 grams of bromine dissolved in $600 \mathrm{cc}$. of ether. ${ }^{1}$ The solution of styrolene was placed in an open beaker surrounded by ice water and it was kept in constant motion by a mechanical stirrer. The rate of flow of the bromine solution was regulated by the discharge of color, from red to a very light yellow. The whole operation was most advantageously carried on in direct sunlight. The crude product obtained by distilling the ether and subsequently crystallizing from dilute alcohol gave a yield of I2 I grams (theory $=\mathrm{I} 23$ grams, therefore $98 \%$ ). The compound obtained by this method was absolutely free of $\beta$-bromostyrolene and was identical in every respect with the product obtained by the experiments of Zincke with ethylbenzene and bromine, which were repeated by us with smaller quantities of materials. In the many experiments performed it was necessary to redistil the styrolene, owing to its great tendency to form solid $m$-styrolene. This operation was accompanied by practically no loss of material.

Preparation of Styrolene Diacetate.-Instead of attempting to prepare styrolene alcohol directly from styrolene dibromide, it was found more convenient first to prepare styrolene diacetate from styrolene dibromide. After many experiments were performed to obtain the highest possible yield of product, the following procedure was found to be the most satisfactory. A mixture of 25 grams (I mol.) of styrolene dibromide and 46.3 grams (I.5 mols.) of carefully fused lead acetate was brought into solution by gently warming in I5O grams of glacial acetic acid. After solution had been effected the reaction mixture was heated very cautiously on an oil bath to a temperature of $120^{\circ}$, at which point the lead bromide began to precipitate in large quantities. Precipitation having ceased at $125^{\circ}$, to which temperature the mixture had been gradually raised, I 5.4 grams ( $0.5 \mathrm{~mol}$ ) ) more of fused lead acetate were added and the heating continued for $2-3$ hours in order to insure completeness of reaction. The greater portion of the glacial acetic acid obtained by filtration of the lead bromide was distilled at diminished pressure. The resulting solution was diluted with water and extracted twelve times with ether. After removing the acetic acid from the combined ether extracts with dilute sodium carbonate, washing once with water, drying with anhydrous sodium sulfate and evaporating, an almost colorless, light yellow oil, weighing 20.4 grams (theory $=2 \mathrm{I}$ grams, therefore 97\%) was obtained. This compound was identical in every respect with the one which $Z$ incke prepared by the action of acetic anhydride on

${ }^{1}$ Compare Tiffeneau, Compt, rend., I45, 8II. 
styrolene alcohol. ${ }^{1}$ It boiled at $159.5^{\circ}$ at $18 \mathrm{~mm}$., $140^{\circ}$ at $\mathrm{I} 2 \mathrm{~mm}$. Zincke found that styrolene diacetate boiled at $274^{\circ}$ at $755 \mathrm{~mm}$, at $183^{\circ}$ to $185^{\circ}$ at $25 \mathrm{mmm}$.

Preparation of Styrolene Alcohol or Phenyl Glycol.-In his attempts to prepare styrolene alcohol, Zincke ${ }^{2}$ used the following reagents with styrolene dibromide: potassium acetate and alcohol; silver acetate and alcohol; the same reagents in acetic acid; silver benzoate in alcohol, in toluene, and in xylene; and potassium carbonate in water. The last one proved to be the most satisfactory, giving a yield of $5-35 \%$ of the theory. He found the main difficulty with the above reagents to be the formation of large quantities of tar and also variable amounts of $\beta$-bromostyrolene, which had to be removed by distillation with steam. Furthermore, he found that the styrolene alcohol contained some of the unchanged styrolene dibromide and also non-hydrolyzed styrolene diacetate. An effort was made by us to obtain styrolene alcohol by the use of styrolene dibromide, potassium formate and methyl alcohol, the mixture being heated in a sealed tube to $100^{\circ}$, but the reaction was incomplete. ${ }^{3}$. A repetition of Zincke's experiment ${ }^{4}$ with styrolene dibromide and aqueous potassium carbonate gave a yield of only $47 \%$ even after three days' boiling. The hydrolysis of styrolene diacetate, obtained in the above experiment, was attempted with dilute sulfuric acid (64\% yield) and with calcium carbonate and water ( $74 \%$ yield). The reaction finally selected was the hydrolysis of styrolene diacetate by means of aqueous potassium carbonate.

To a solution of 18.9 grams ( 1.5 mols.) of potassium carbonate in $500 \mathrm{cc}$. of water were added 20.4 grams ( 1 mol.) of pure styrolene diacetate. The reaction mixture was kept at the boiling point for 6 hours under a reverse condenser and then was quickly filtered. After distilling off from one-third to one-half of the water at diminished pressure (18-25 $\mathrm{mm}$ ) the remaining solution was treated with solid potassium carbonate to saturation. By this procedure a large portion of the styrolene alcohol precipitated and after filtration the filtrate was extracted eight times with ether. The combined extracts after drying with anhydrous sodium sulfate were treated with an equal volume of ligroin (b. p. $60-80^{\circ}$ ). By distilling the solution to the point of turbidity, the remaining styrolene alcohol crystallized out on cooling. A theoretical yield of I3.I grams of the crude product was obtained. By several crystallizations from a mixture of ether and ligroin, a final yield of II.I grams (theory $=$ I3.I grams, therefore $90 \%$ ) of pure product was obtained, corre-

${ }^{1}$ Ann., 216, 295.

2Ibid., 2I6, 288.

${ }^{3}$ Rec. Pay-Bas, 22, I16; 23, 343; Chem. Zentr., I902, II, 928; compare Nef, Ann., 335, 260.

Loc. cit. 
sponding in every way with the product obtained by the repetition of Zincke's experiments. By the methods outlined above, a yield of $86 \%$ of styrolene alcohol was obtained when calculated to the styrolene used. On the same basis, Zincke obtained $30 \%$. The experiment described here to illustrate the preparation of styrolene alcohol was repeated a great many times during the progress of this work. Frequently, larger quantities of materials were used, but in all cases the same general result was obtained.

Styrolene Alcohol and Potassium Permanganate.-It has been shown previously that benzoylcarbinol and benzoylformaldehyde ${ }^{1}$ are oxidized by potassium permanganate to benzoic acid exclusively, no trace of mandelic or benzoylformic acids being obtained. That the same result is obtained with styrolene alcohol is shown by the following experiment: A solution of 2 grams ( $\mathrm{I}$ mol.) of styrolene alcohol in $48 \mathrm{cc}$. of water was added slowly to 9.14 grams (I.5 mols.) of potassium permanganate dissolved in $370 \mathrm{cc}$. of water. The reaction mixture was kept at $0^{\circ}$ for I 2-I 5 hours, after which the oxide of manganese was filtered and washed. The filtrate was acidulated with dilute sulfuric acid, and then extracted six times with ether. After drying the combined ether extracts with anhydrous sodium sulfate, and evaporating, a residue of 1.76 grams of benzoic acid was obtained (theory $=1.76$ grams). Similar studies were made with benzaldehyde, benzyl alcohol, methyl alcohol and formaldehyde. (a) In the same manner, therefore, I.53 grams of benzaldehyde (free of benzoic acid) were added a drop at a time to 9.14 grams (I.5 mols.) of potassium permanganate dissolved in $4 \mathrm{r} 8 \mathrm{cc}$. of water. After working up the reaction mixture in the usual manner, 1.61 grams of benzoic acid were obtained (theory $=1.76$ grams, therefore $91.4 \%$ ). (b) An equivalent weight of benzyl alcohol, i. e., 1.56 grams, in $48 \mathrm{cc}$. of water was added to 9.14 grams ( $1.5 \mathrm{mols}$.) of potassium permanganate dissolved in $370 \mathrm{cc}$. of water as above. A final product of 1.69 grams of benzoic acid was obtained (i.e., $96 \%$ ). In the experiments noted here $(a)$ and $(b)$, the excess of potassium permanganate was reduced with the least amount of ethyl alcohol, before working up the reaction mixture. The temperature in experiments $(a)$ and $(b)$ was maintained at $10^{\circ}$. Special experiments confirmed the well known action of potassium permanganate on methyl alcohol and formaldehyde. From these results, it is evident that the oxidation of styrolene alcohol with potassium permanganate in the absence of alkali, may follow any of the five possibilities.

Styrolene Alcohol, Potassium Permanganate and Sodium Hydroxide.Since benzoylcarbinol, benzoylformaldehyde and mandelic acid are all oxidized by alkalin potassium permanganate to give benzoylformic ${ }^{1}$ Am. Chem. J., 35, II 5, 128. 
acid, it became important to ascertain whether this acid was a reaction product of styrolene alcohol with the same reagents. To test this point the following experiment was performed: To 8.9I grams ( $1.5 \mathrm{mols}$.) of potassium permanganate and 2.8 grams ( 5 mols.) of sodium hydroxide dissolved in $375 \mathrm{cc}$. of water were slowly added 1.95 grams (I mol.) of styrolene alcohol dissolved in $50 \mathrm{cc}$. of water. The temperature of the reaction mixture was maintained at $4^{\circ}$. After standing over night, the solution was worked up in the usual manner. A yield of 1.63 grams of benzoic acid (theory $=1.72$ grams, therefore $94.9 \%$ ) was obtained. It is to be concluded, therefore, from this result that the oxidation reaction of styrolene alcohol under these conditions does not proceed in accordance with equations (III), (IV) or (V). Consequently, the action of alkalin potassium permanganate on benzaldehyde and benzyl alcohol was studied. To a solution of 9.23 grams ( 1.5 mols.) of potassium permanganate and 2.9 grams ( 5 mols.) of sodium hydroxide in $425 \mathrm{cc}$. of water were added 1.54 grams of pure benzaldehyde. After standing over night at $6^{\circ}$, a yield of 1.63 grams of benzoic acid was obtained (theory $=1.77$ grams, therefore $92 \%$ ). That benzyl alcohol reacts in the same manner was shown by the following experiment: To a solution of 9.28 grams of potassium permanganate ( $1.5 \mathrm{mols}$.) and 2.9 grams ( $5 \mathrm{mols}$.) of sodium hydroxide in $430 \mathrm{cc}$. of water were added 1.58 grams ( $\mathrm{I}$ mol.) of benzyl alcohol. After standing over night at $10^{\circ}, 1.75$ grams of benzoic acid were obtained (theory $=1.79$ grams, therefore $98 \%$ ). An examination of the behavior of methyl alcohol and formaldehyde towards alkalin potassium permanganate would evidently lead to a choice between equations (I) and (II). In view of the fact that the excess of potassium permanganate used was reduced by a few drops of ethyl alcohol in the general procedure employed, a little less than the calculated amount of potassium permanganate was used in this experiment. To a solution of I0.64 grams of potassium permanganate ( 2 mols.) and 8 grams of potassium hydroxide ( 2 mols.) in $500 \mathrm{cc}$. of water, were added I.I 5 grams of pure methyl alcohol. Oxidation began at once. After the reaction mixture had been worked up as 1sual, I.45 grams of carbon dioxide were obtained (theory $=1.47$, therefore $98.6 \%$ ). Therefore, it may be concluded that methyl alcohol under these experimental conditions is oxidized quantitatively to carbon dioxide, since the weight of potassium permanganate equivalent to I.I 5 grams of methyl alcohol is I I.34 grams. It follows, therefore, that formaldehyde is oxidized quantitatively by alkalin permanganate to carbon dioxide. This well known fact was verified with x.08 gratns of formaldehyde. Frankforter and West ${ }^{1}$ have shown that hydrogen is obtained quantitatively as one of the products of reaction when formaldehyde is acted on by hydrogen peroxide in alkalin solution.

${ }^{3}$ ThIS JoUrnal, 27, 7 I4. 
Heimrod and Levee ${ }^{1}$ have confirmed this result, and have assumed that the evolution of hydrogen is to be considered as an evidence of the intermediate formation of formaldehyde in the oxidation of certain compounds.

From this group of experiments it is at once obvious that the oxidation of styrolene alcohol by means of alkalin potassium permanganate must take place in accordance with equations (I) or (II) and that, in effect, equations (III), (IV) and (V) are excluded on the grounds that benzoylformic acid is not formed as a reaction product. If mandelic aldehyde is formed in this reaction it is to be expected, a priori, that it would be oxidized to mandelic acid and this in turn to benzoylformic acid. Mandelic acid, like lactic acid, ${ }^{2}$ will oxidize to the corresponding keto-acid. ${ }^{3}$

Styrolene Alcohol, Potassium Ferricyanide and Potassium Hydroxide.Since benzoylcarbinol and benzoylformaldehyde are both oxidized to mandelic ácid' with potassium ferricyanide, ${ }^{4}$ it was obviously of very great interest to ascertain the action of this reagent on styrolene alcohol. If styrolene alcohol, in the presence of alkalies, is dissociated in accordance with equations (III), (IV) or (V), then it becomes perfectly evident that mandelic acid should be one of the oxidation products. If this acid is absent, then these possibilities are rigidly excluded from consideration. A great many experiments carried out in this direction proved that mandelic acid was absent. In all cases, benzoic acid was shown to be the main reaction product. To 51.6 grams $(3.5 \mathrm{mols}$.) of potassium ferricyanide and 20.8 grams ( 5 mols.) of potassium hydroxicle dissolved in $150 \mathrm{cc}$. of water, were added 2 grams ( $\mathrm{r}$ mol.) of styrolene alcohol dissolved in $50 \mathrm{cc}$. of water. The reaction mixture was allowed to stand over night at $10^{\circ}$. After working up in the usual manner, 1.76 grams (I00\%) of benzoic acid were obtained. According to. Nef, the metallic salts of the alcohols dissociate with much greater ease than the free substance. For this reason, it was supposed that increasing the concentration of the alkali would bring about a more ready dissociation of styrolene alcohol in accordance with equations (III), (IV) or (V), and thus it was thought that mandelic acid would be formed as a reaction product. In every case, however, benzoic acid was found as the main oxidation product. The increase in the concentration of potassium hydroxide was carried to the point where potassium ferrocyanide began to separate out in characteristically large yellow crystals. On the other hand, in a number of experiments carried on under the conditions just stated, a small quantity of benzaldehyde was formed. Its identity was established by its phenylhydrazone. As indicated above, Zincke found

\footnotetext{
${ }^{1}$ Biochem. Z., 29, 3 I.

${ }^{2}$ Ber., I7, 840 .

${ }^{8}$ Am. Chem. J., 35, 130.

${ }^{4}$ Ibid., 35, 127.
} 
that benzaldehyde was formed quantitatively from strrolene alcohol when treated with certain oxidizing agents

Since mandelic acid was not found as a reaction product, it became necessary to study the behavion of benzaldehyde and benzyl alcohol towards potassium ferricyanide under the conditions employed above. To a solution of 51.9 grams $(3.5 \mathrm{mols}$ ) of potassium ferricyanide and 20.9 grams $(5$ mols.) of potassium hydroxide in 200 cc. of water, 1.53 grams of benzaldehyde were slowly added. When treated in the usual manner, 1.64 grams (theory $=1.76$ grams, therefore $93 \%$ ) of benzoic acid were obtained. When I.5 I grams of benzyl alcohol were treated with an equivalent amount of the same reagents and at the same temperature (10\%), 1.67 grams of benzoic acid were obtained (theory $=\mathrm{I} .7 \mathrm{I}$ grams, therefore $97 \%$ ). When an amount of potassium mandelate equivalent to the styrolene alcohol used was oxidized with alkalin potassimu ferricyanide under the above conditions, 0.30 gram of benzoic acid was obtained, the remainder being unchanged mandelic acid. ${ }^{1}$ Since styrolene alcohol, benzaldehyde and benzyl alcohol are oxidized to benzoic acid with potassium ferricyanide and since, furthermore, mandelic acid is not found as a reaction product with the former, it is perfectly obvious that, in effect, equations (III), (IV) and (V) are excluded. Had mandelic aldehyde forned as an intermediate product, it would have been expected, a prior, to be oxidized to mandelic acid, and not to dissociate into benzaldehyde and formaldehyde, $i$.

$$
\mathrm{C}_{i} \mathrm{H}_{5}, \mathrm{CHOH} . \mathrm{CHO} \rightarrow \mathrm{C}_{6} \mathrm{H}_{5} \cdot \mathrm{CHO}+\mathrm{H}_{2} \mathrm{CO}
$$

It was found that methyl alcohol and formaldehyde reduced alkalin potassium ferricyanide solutions. ${ }^{2}$

Styrolene Alcohol, Silier Oxide and Potassium Hydroxide.-The action of silver oxide towards styrolene alcohol has not been previously studied. When the oxidation is carried on either in the absence or presence of alkalies, one of the products common to both reactions is benzoic acid. A solution of 2 grams of styrolene alcohol in $150 \mathrm{cc}$. of water was added to a mixture of the silver oxide obtained from 30 grams of silver nitrate and 3.2 grams of potassium hydroxide in $50 \mathrm{cc}$. of water. The reaction mixture was heated to a temperature of $60^{\circ}$ (oil bath), under a reverse condenser. At the end of this time, the silver oxide and reduced silver were filtered and washed with hot water, until the washings were free of alkali and silver salts. The residue was washed back to a flask and then cautiously treated with dilute ammonium hydroxide. After washing by decantation, and repeating the treatment with ammonium hydroxide until the washings were free of soluble silver compounds, the metallic siver was tinally transferred to the paper and washed with hot water

\footnotetext{
:Compare Am. Chem. J., 35, г2

"Compare heintod and Levee, Biochem. Z., 29, 37 .
} 
until the washings were neutral. After drying, the residue weighed 8.75 grams (theory for the equivalent of three atoms of consumed oxygen $=$ 9.39 grams, therefore $93.2 \%$ ). In a duplicate experiment, the silver weighed 9.20 grams (therefore $98 \%$ ).

One-half of the aqueous filtrate, after diluting to $1000 \mathrm{cc}$. was acidulated with dilute sulfuric acid and extracted five times with ether. After drying the combined ether extracts, evaporating and weighing the residue, 0.88 gram of benzoic acid was obtained, or a total of 1.76 grams (therefore $100 \%$ ). This result was duplicated in an experiment in which 4 grams of styrolene alcohol had been used, i. e., 3.52 grams of benzoic acid were obtained. This residue was found to be completely distilled by steam, thus showing mandelic acid to be absent. ${ }^{1}$

An examination of an aliquot part of the above aqueous filtrate for formic acid by Jones's method showed 0.68 gram (theory $=0.69$ gram, therefore $98.5 \%$ ) to be present. Another portion of the filtrate was examined for carbon dioxide by means of the Foulk apparatus ${ }^{2}$ with a negative result.

From these results and a consideration of the facts given below it will be seen that, in effect, benzoylcarbinol is the first oxidation product of styrolene alcohol with silver oxide in the presence of potassium hydroxide. That the oxidation reaction could not have taken place in accordance with equation (I) is shown by the following experiments: (I) (a) After a mixture of 2.3 grams (4 mols.) of sodium hydroxide dissolved in $200 \mathrm{cc}$. of water, the well washed silver oxide from 20 grams of silver nitrate, and I.498 grams (the equivalent of 2 grams of styrolene alcohol) of pure benzaldehyde had been heated to $60^{\circ}$ (oil bath) for 36 hours, a yield of 1.6I4 grams of benzoic acid was obtained (theory $=1.72$ grams, therefore $93 \%)$. (b) Furthermore, a mixture of 0.46 gram of methyl alcohol, 2.3 grams of sodium hydroxide dissolved in $200 \mathrm{cc}$. of water, and the silver oxide from 20 grams of silver nitrate, was treated in the same manner. Only a trace of metallic silver was obtained.

(2) (a) A study of benzyl alcohol was made under the same conditions as with betizaldehyde, the weight of the benzyl alcohol being I.49 grams (duplicate $=1.55$ grams). A yield of 0.62 gram ( 0.69 gram) of benzoic acid (theory $=1.76$ grams, therefore $35.2 \%$ ) and 1.27 grams ( 1.32 grams) of silver was obtained (theory for silver equivalent to 2 atoms of oxygen $=$ 6.0 grams). A residue of 0.86 gram ( 0.89 gram) of unchanged benzyl alcohol was obtained. (b) A special experiment was made with a solution containing 0.46 gram of formaldehyde, which was added to the same reagents as employed in $2(a)$. The products of the reaction were formic acid $(86.15 \%)$ and carbon dioxide $(7.14 \%)$ and 3.53 grams of silver.

${ }^{1}$ Compare Nef, Ann., 335, 3 I 4.

2 Foulk, "Notes on Quantitative Chemical Analysis," p. 221. 
These results are in accord with the well known behavior of formaldehyde. It therefore becomes apparent from the result of (2) (a) that equation (II) is also excluded as an expression of one of the oxidation stages of styrolene alcohol.

(3) That benzoylformaldehyde is not formed as an intermediate product is evident from the fact that it has been shown previously ${ }^{1}$ that this substance is oxidized with silver oxide in the presence of alkali, to benzoic acid and carbon dioxide as the main reaction products, together with a trace of mandelic acid. That is, a greater portion of the benzoylformaldehyde dissociates into benzaldehyde and carbon monoxide,

$$
\mathrm{C}_{6} \mathrm{H}_{5} \cdot \mathrm{CO} . \mathrm{CHO} \longrightarrow \mathrm{C}_{8} \mathrm{H}_{5} \cdot \mathrm{CHO}+\mathrm{CO} \text {, }
$$

and these two compounds are subsequently oxidized to benzoic acid and carbon dioxide, respectively. Since carbon dioxide and mandelic acid are not found as reaction products in the oxidation of styrolene alcohol with silver oxide in the presence of alkali, it is to be concluded, therefore, that the formation of benzoylformaldehyde as an intermediate product is rigidly excluded.

(4) In his studies with mandelic aldehyde acetate and lactic aldehyde acetate $^{2}$ Nef found that these substances when treated with water at $100^{\circ}$ were hydrolyzed, and subsequently rearranged into benzoylcarbinol and acetol; respectively. Therefore, it becomes apparent that had mandelic aldehyde formed in the above experiment with styrolene alcohol, it would have been converted immediately to benzoylcarbinol. Furthermore, it is highly probable that some of the mandelic aldehyde formed would have been oxidized to mandelic acid. It is worthy of note that sodium mandelate is stable towards silver oxide in the presence of alkalies, even at $100 \%$ If mandelic aldehyde was formed momentarily, it might have been dissociated into benzaldehyde and formaldehyde,

$$
\mathrm{C}_{6} \mathrm{H}_{5} . \mathrm{CHOH} . \mathrm{CHO} \rightarrow \mathrm{C}_{6} \mathrm{H}_{3} \cdot \mathrm{CHO}+\mathrm{H}_{2} \mathrm{CO} \text {, }
$$

and these compotinds then would be oxidized to their respective acids. From these considerations, it is seen that mandelic aldehyde remains a theoretical possibility as an intermediate oxidation product in the oxidation of styrolene alcohol with silver oxide in the presence of alkali. ${ }^{3}$

(5) It has been previously shown by one of us that benzoylcarbinol is oxidized to benzoic acid with silver oxide in the presence of alkali. The silver obtained in this experiment (I2.6 grams) was exactly equivalent to two atoms of consumed oxygen. Therefore, it is apparent at once that benzoylcarbinol is dissociated in this reaction to benzaldehyde and formaldehyde,

\footnotetext{
${ }^{1}$ Am. Chem. I., 35, 131.

${ }^{2}$ Ann., 335, 266.

' Compare Zincke, Ann., 216, 309.

- Compare Nef, Ann., 335, 274.
} 


$$
\mathrm{C}_{8} \mathrm{H}_{6} \cdot \mathrm{CO} \cdot \mathrm{CH}_{2} \mathrm{OH} \rightarrow \mathrm{C}_{8} \mathrm{H}_{5} \cdot \mathrm{CHO}+\mathrm{H}_{2} \mathrm{CO}(\mathrm{CHOH}) \text {, }
$$

and these two aldehydes are then subsequently oxidized to benzoic and formic acids, respectively, i. e.,.

$$
\mathrm{C}_{8} \mathrm{H}_{5} \cdot \mathrm{CO} \cdot \mathrm{CH}_{2} \mathrm{OH}+2 \mathrm{Ag}_{2} \mathrm{O} \longrightarrow \mathrm{C}_{8} \mathrm{H}_{5} \cdot \mathrm{COOH}+\mathrm{HCOOH}+4 \mathrm{Ag} \text {. }
$$

It is to be observed that had the benzoylcarbinol molecule formed in this reaction been oxidized at the primary alcohol group, benzoylformaldehyde would have been formed. This aldehyde as indicated in (3) would have been oxidized to benzoic acid and carbon dioxide, with a trace of mandelic acid present. To accomplish this oxidation of benzoylcarbinol would require three atoms of oxygen instead of two. As above noted, sodium mandelate is stable at $100^{\circ}$ in the presence of silver oxide and alkalies. The results of the experiments enumerated here are decisive proof of the dissociation of the benzoylcarbinol molecule. In this same connection it has been shown that benzoylcarbinol is oxidized with silver oxide in the absence of alkali to benzoic acid and carbon dioxide. The weight of the silver obtained in this latter experiment was equivalent to three atoms of consumed oxygen,

$$
\mathrm{C}_{6} \mathrm{H}_{5} \cdot \mathrm{CO} \cdot \mathrm{CH}_{2} \mathrm{OH}+{ }_{3} \mathrm{Ag}_{2} \mathrm{O} \longrightarrow \mathrm{C}_{6} \mathrm{H}_{5} \cdot \mathrm{COOH}+\mathrm{H}_{2} \mathrm{O}+\mathrm{CO}_{2}+6 \mathrm{Ag} \text {. }
$$

In order to prove the actual presence of formic acid in the oxidation of benzoylcarbinol, the following experiment was carried through: A mixture of 2 grams of benzoylcarbinol, the silver oxide from 20 grams of silver nitrate, and 3.2 grams of potassium hydroxide, was treated and worked up in the usual manner. The products of reaction were: silver 6.42 grams (the silver equivalent to two atoms of consumed oxygen $=$ 6.35 grams); formic acid 0.68 gram (theory $=0.68 \mathrm{gram}$ ); benzoic acid from one-half the original filtrate 0.90 gram or a total of I.80 grams (theory $=1.80$ grams). Carbon dioxide was not present.

Finally, since in the oxidation of both styrolene alcohol and benzoylcarbinol with silver oxide in the presence of alkali, benzoic acid and formic acid are obtained, and carbon dioxide is absent and since the weight of the silver obtained from the styrolene alcohol reaction is just equivalent to one more atom of oxygen (i. e., three) than that obtained in the oxidation of benzoylcarbinol (i.e., two) it is to be concluded that in the oxidation of styrolene alcohol under the conditions employed above, benzoylcarbinol is the first oxidation product. That is, equation (III) expresses the course of the oxidation reaction, $i . e$. , in steps.

(a) $\mathrm{C}_{6} \mathrm{H}_{8} \cdot \mathrm{CHOH} \cdot \mathrm{CH}_{2} \mathrm{OH}+\mathrm{Ag}_{2} \mathrm{O} \longrightarrow \mathrm{C}_{6} \mathrm{H}_{5} \cdot \mathrm{CO} \cdot \mathrm{CH}_{2} \mathrm{OH}+\mathrm{H}_{2} \mathrm{O}+2 \mathrm{Ag}$

(b) $\mathrm{C}_{6} \mathrm{H}_{5} \cdot \mathrm{CO} \cdot \mathrm{CH}_{2} \mathrm{OH} \longrightarrow \mathrm{C}_{6} \mathrm{H}_{5} \cdot \mathrm{CHO}+\mathrm{H}_{2} \mathrm{CO}$

(c) $\mathrm{C}_{6} \mathrm{H}_{5} \cdot \mathrm{CHO}+\mathrm{H}_{2} \mathrm{CO}+2 \mathrm{Ag}_{2} \mathrm{O} \longrightarrow \mathrm{C}_{6} \mathrm{H}_{5} \cdot \mathrm{COOH}+\mathrm{H} \cdot \mathrm{COOH}+4 \mathrm{Ag}$ or, finally 


\section{$\mathrm{C}_{6} \mathrm{H}_{5} \cdot \mathrm{CHOH} \cdot \mathrm{CH}_{2} \mathrm{OH}+3 \mathrm{Ag}_{2} \mathrm{O} \longrightarrow \mathrm{C}_{4} \mathrm{H}_{5} \cdot \mathrm{COOH}+\mathrm{H} \cdot \mathrm{COOH}+6 \mathrm{Ag}$.}

Styrolene Alcohol and Silver Oxide.-A study of the behavior of styrolene towards silver oxide, in the absence of alkali, was made in order to ascertain whether the course of the reaction is the same as in the presence of alkali. To the well washed silver oxide, obtained from 30 grams of silver nitrate, suspended in $15 \circ \mathrm{cc}$. of water, was added a solution of 2 grams of styrolene alcohol dissolved in $50 \mathrm{cc}$. of water. While standing at room temperature for several days, reduction took place with an evolution of carbon dioxide. After the reaction mixture had been worked up in the usual manner, 1.42 grams of benzoic acid were obtained (theory 1.76 grams, therefore $80 \%$ ) and I 1.75 grams of silver (theory for silver equivalent to four atoms of oxygen $=12.52$ grams, therefore $93.8 \%$ ). It is at once apparent that this reaction differs from the one carried out in the presence of alkali, in that the oxygen consumed is equivalent to four atoms instead of three, and also, in that carbon dioxide is evolved and formic acid is absent. An account of the studies made on the possible dissociation products of styrolene alcohol in the presence of silver oxide will be given briefly: (a) When benzaldehyde ( $\mathrm{I} .54$ grams) was acted on by the same reagents and in the same amounts as styrolene alcohol, $x .66$ grams of benzoic acid were obtained (i. e., $9 \mathrm{I} \%$ ). When a mixture of $x .15$ grams of methyl alcohol dissolved in $500 \mathrm{cc}$. of water and the silver oxide from $5^{\circ}$ grams of silver nitrate stood at room temperature for several days, no metallic silver was obtained, thus showing that no oxidation had taken place. (b) When benzyl alcohol ( 1.46 grams) was treated in the same manner as benzaldehyde, no reduction of the silver oxide took place. This result was repeatedly obtained. As was to have been expected, 0.43 gram of formaldehyde was oxidized to carbon dioxide, the metallic silver obtained showing the oxidation to have been complete. An examination of the results of these experiments shows conclusively that the oxidation of styrolene alcohol with silver oxide, in the absence of alkali, cannot proceed in accordance with equations (I) and (II).

(c) Since benzoylformaldehyde hydrate is unacted on by silver oxide at room temperature, ${ }^{1}$ and since this aldehyde was not obtained in the above experiments, it is at once apparent that equation (V) is also excluded.

(d) In view of the experiments of Wohl and Lange ${ }^{2}$ and also more recently those of $\mathrm{Kranz}^{3}$ with lactic aldehyde, it is hardly probable that mandelic aldehyde would rearrange to benzoylcarbinol at the temperature employed in this series of experiments. Had it formed, it would

Am. Chem. I., 35, I31.

- Ber., 41, 3612.

"Chemicke Listy, 5, 323-7; C. A., 6, 739 . 
have been either oxidized to mandelic acid, or it would have been dissociated in accordance with the following equation:

$$
\mathrm{C}_{8} \mathrm{H}_{5} \text {. } \mathrm{CHOH} . \mathrm{CHO} \longrightarrow \mathrm{C}_{8} \mathrm{H}_{5} . \mathrm{CHO}+\mathrm{H}_{2} \mathrm{CO} \text {, }
$$

and then these aldehydes would undergo oxidation. Although mandelic acid was not found as an oxidation product in this series, yet mandelic aldehyde remains as a theoretical possibility. In view of the results of the action of alkalin bromine solutions on styrolene alcohol, it is very improbable that mandelic aldehyde is actually formed in the oxidation of styrolene alcohol with silver oxide in the absence of alkali. (e) Finally, that benzoylcarbinol is oxidized by silver oxide in the absence of alkali to give benzoic acid and carbon dioxide, is shown by the following experiment: A mixture of 2 grams of benzoylcarbinol, the silver oxide from 20 grams of silver nitrate, and $200 \mathrm{cc}$. of water was subjected to the same treatment as styrolene alcohol. The following reaction products were obtained: benzoic acid, I.80 grams (theory $=1.80$ grams); silver, 6.37 grams (theory $=6.52$ grams, therefore $97.7 \%$ ); carbon dioxide, 0.22 gram, from 0.96 gram of barium carbonate (theory $=0.65$ gram, therefore $33 \%$ ). The metallic silver obtained was equivalent to three atoms of consumed oxygen, while with styrolene alcohol the silver obtained was equivalent to four atoms of consumed oxygen. Without any further discussion, it must now be concluded that styrolene alcohol is oxidized with silver oxide in the absence of alkali in accordance with the following equations:

(a) $\mathrm{C}_{6} \mathrm{H}_{5} \cdot \mathrm{CHOH} \cdot \mathrm{CH}_{2} \mathrm{OH}+\mathrm{Ag}_{2} \mathrm{O} \longrightarrow \mathrm{C}_{6} \mathrm{H}_{5} \cdot \mathrm{CO} \cdot \mathrm{CH}_{2} \mathrm{OH}+\mathrm{H}_{2} \mathrm{O}+2 \mathrm{Ag}$

(b) $\mathrm{C}_{6} \mathrm{H}_{5} \cdot \mathrm{CO} \cdot \mathrm{CH}_{2} \mathrm{OH} \longrightarrow \mathrm{C}_{8} \mathrm{H}_{5} \cdot \mathrm{CHO}+\mathrm{H}_{2} \mathrm{CO}$

(c) $\mathrm{C}_{8} \mathrm{H}_{5} \cdot \mathrm{CHO}+\mathrm{H}_{2} \mathrm{CO}+3 \mathrm{Ag}_{2} \mathrm{O} \longrightarrow \mathrm{C}_{6} \mathrm{H}_{5} \cdot \mathrm{COOH}+\mathrm{H}_{2} \mathrm{O}+\mathrm{CO}_{2}+6 \mathrm{Ag}$, or, finally,

$\mathrm{C}_{6} \mathrm{H}_{5} \cdot \mathrm{CHOH} \cdot \mathrm{CH}_{2} \mathrm{OH}+{ }_{4} \mathrm{Ag}_{2} \mathrm{O} \longrightarrow \mathrm{C}_{8} \mathrm{H}_{5} \mathrm{COOH}+\mathrm{CO}_{2}+{ }_{2} \mathrm{H}_{2} \mathrm{O}+8 \mathrm{Ag}$, that is, benzoylcarbinol is the intermediate oxidation product in this reaction. That the dissociation indicated in $(b)$ takes place is certain because, had an oxidation of the primary alcohol group taken place, benzoylformaldehyde would have been formed. This substance as above noted is stable under the conditions of these experiments. The hydrolytic splitting of benzoylcarbinol, i. e.,

$$
\mathrm{C}_{6} \mathrm{H}_{5} \cdot \mathrm{CO} \cdot \mathrm{CH}_{2} \mathrm{OH}+\mathrm{HOH} \longrightarrow \mathrm{C}_{6} \mathrm{H}_{5} \mathrm{COOH}+\mathrm{CH}_{3} \mathrm{OH} \text {, }
$$

does not take place, since methyl alcohol is stable towards silver oxide under the conditions of these experiments. Hence the presence of carbon dioxide would remain to be explained.

Styrolene Alcohol, Bromine and Potassium Carbonate.-Zincke ${ }^{1}$ found that benzoylcarbinol could be obtained by the oxidation of styrolene

1 Ann., 216, 307. 
alcohol with nitric acid. Fischer and Tafel $^{1}$ found that glycerol was oxidized with bromine in alkalin soltution to glyceric aldehyde and dihydroxyacetone. It was thought that alkalin bromine solutions might react in the same manner on styrolene alcohol to give benzoylcarbinol and mandelic aldehyde. Two grams of styrolene alcohol, dissolved in water, were added to a solution of 35 grams of potassium carbonate and I5 grams of bromine, the total volume of the reaction mixture being $100 \mathrm{cc}$. After standing over night, the reaction mixture yielded $0.8 \mathrm{I}$ gram of benzoylcarbinol (theory $=1.97$ grams, therefore $4 \mathrm{I} .1 \%$ ), which was identical in every respect with a specimen prepared by the hydrolysis of acetophenone acetate. ${ }^{2}$ In this experiment, benzoic acid and an oil which hardened to a viscous mass were also obtained. This reaction is to be studied further.

Solutions of copper nitrate, sulfate and acetate have no effect on styrolene alcohol, even when the reaction mixtures are kept at $100^{\circ}$.

In conclusion, we wish to thank Mr. C. R. Parkinson of this laboratory for valuable assistance rendered during the progress of this work.

Conumaus, ono.

[CONTRIBUTION FROM THE ChEMICAL LABORATORY OF THE UNIVERSTTY OF MICHGAN.]

STUDIES IN THE CYCLOPENTADIENE SERIES. II.

2,3-DIBENZOYL-5-NITROCYCLOPENTADIENE. ${ }^{3}$

BY WILliam J. Hale and LaMbert Thorp.

Received November 16, 1912.

The condensation of acetonylacetone with the 1,3-dialdehyde, nitromalonic aldehyde, has been shown to give a 2,3-diacetyl-5-nitrocyclopentadiene. $^{*}$ The presence of acetone nuclei in this diketone makes possible also the formation of phenol derivatives during the course of the reaction, ${ }^{5}$ but the proportion of the latter $(20 \%)$ is not large in comparison with the cyclopentadiene produced.

In order to favor only the cyclopentadiene condensation it became necessary, therefore, to employ a diketone of similar structure to acetonylacetone but one containing aryl groups in place of the two active methyl groups of the latter. As the simplest example of this 1,4 -diketone class we have taken diphenacyl, $\mathrm{C}_{6} \mathrm{H}_{5} \cdot \mathrm{CO} \cdot \mathrm{CH}_{2} \cdot \mathrm{CH}_{2} \cdot \mathrm{CO} \cdot \mathrm{C}_{6} \mathrm{H}_{5}$. This ketone, however, has the disadvantage of being only slightly soluble in an aqueousalcoholic solution, the medium of these condensations. Acetonylacetone, on the other hand, is very soluble in this medium. Consequently a com-

'Ber, 20, 3385.

'Am. Chem. J., 35, 120.

3 The work described in this article formed part of a thesis submitted for the degree of Doctor of Philosophy in the University of Michigan by Lambert Thorp.

Ber., 45, I596 (I9I2); ThIS JoURNAL, 34, 1580 (1912).

s Am. Chem.J., 39, 680 (IgO8). 